\title{
Prestroke Antiplatelet Effect on Symptomatic Intracranial Hemorrhage and Functional Outcome in Intravenous Thrombolysis
}

\author{
Jay Chol Choi, ${ }^{\mathrm{a}^{*}}$ Ji Sung Lee, ${ }^{\mathrm{b}^{*}}$ Tai Hwan Park, ${ }^{\mathrm{c}}$ Yong-Jin Cho, ${ }^{\mathrm{d}}$ Jong-Moo Park, ${ }^{\mathrm{e}}$ Kyusik Kang,
}

Kyung Bok Lee, ${ }^{\mathrm{f}}$ Soo Joo Lee, ${ }^{\mathrm{g}}$ Jae Guk Kim, ${ }^{\mathrm{g}}$ Jun Lee, ${ }^{\mathrm{h}}$ Man-Seok Park, ${ }^{\mathrm{i}}$ Kang-Ho Choi,, ${ }^{\mathrm{i}}$ Joon-Tae Kim, ${ }^{\mathrm{i}}$ Kyung-Ho Yu, ${ }^{j}$ Byung-Chul Lee, ${ }^{\mathrm{j}}$ Mi-Sun Oh, ${ }^{\mathrm{j}}$ Jae-Kwan Cha, ${ }^{\mathrm{k}}$ Dae-Hyun Kim, ${ }^{\mathrm{k}}$ Hyun-Wook Nah, ${ }^{\mathrm{k}}$ Dong-Eog Kim, ${ }^{\mathrm{l}}$ Wi-Sun Ryu, ${ }^{\mathrm{l}}$ Beom Joon Kim, ${ }^{\mathrm{m}}$ Hee-Joon Bae, ${ }^{\mathrm{m}}$ Wook-Joo Kim, ${ }^{\mathrm{n}}$ Dong-Ick Shin, ${ }^{ }$ Min-Ju Yeo, ${ }^{o}$ Sung Il Sohn, ${ }^{\mathrm{p}}$ Jeong-Ho Hong, ${ }^{\mathrm{p}}$ Juneyoung Lee, ${ }^{\mathrm{q}}$ Keun-Sik Hong ${ }^{\mathrm{d}}$

aDepartment of Neurology, Jeju National University, Jeju, Korea

${ }^{b}$ Clinical Research Center, Asan Medical Center, Seoul, Korea

'Department of Neurology, Seoul Medical Center, Seoul, Korea

dDepartment of Neurology, Ilsan Paik Hospital, Inje University, Goyang, Korea

eDepartment of Neurology, Eulji General Hospital, Eulji University, Seoul, Korea

fDepartment of Neurology, Soonchunhyang University College of Medicine, Seoul, Korea

${ }^{9}$ Department of Neurology, Eulji University Hospital, Daejeon, Korea

hDepartment of Neurology, Yeungnam University Hospital, Daegu, Korea

'Department of Neurology, Chonnam National University Hospital, Gwangju, Korea

'Department of Neurology, Hallym University Sacred Heart Hospital, Anyang, Korea

kDepartment of Neurology, Dong-A University College of Medicine, Busan, Korea

'Department of Neurology, Dongguk University Ilsan Hospital, Goyang, Korea

mDepartment of Neurology, Seoul National University Bundang Hospital, Seoul National University College of Medicine, Seongnam, Korea

"Department of Neurology, Ulsan University Hospital, Ulsan, Korea

'Department of Neurology, Chungbuk National University Hospital, Cheongju, Korea

PDepartment of Neurology, Keimyung University Dongsan Medical Center, Daegu, Korea

"Department of Biostatistics, Korea University College of Medicine, Seoul, Korea

Background and Purpose About $30 \%-40 \%$ of stroke patients are taking antiplatelet at the time of their strokes, which might increase the risk of symptomatic intracranial hemorrhage (SICH) with intravenous tissue plasminogen activator (IV-TPA) therapy. We aimed to assess the effect of prestroke antiplatelet on the SICH risk and functional outcome in Koreans treated with IV-TPA.

Methods From a prospective stroke registry, we identified patients treated with IV-TPA between October 2009 and November 2014. Prestroke antiplatelet use was defined as taking antiplatelet within 7 days before the stroke onset. The primary outcome was SICH. Secondary outcomes were discharge modified Rankin Scale (mRS) score and in-hospital mortality.

Results Of 1,715 patients treated with IV-TPA, 441 (25.7\%) were on prestroke antiplatelet. Prestroke antiplatelet users versus non-users were more likely to be older, to have multiple vascular risk factors. Prestroke antiplatelet use was associated with an increased risk of SICH $(5.9 \%$ vs. 3.0\%; adjusted odds ratio [OR] 1.79 [1.05-3.04]). However, at discharge, the two groups did not differ in mRS distribution (adjusted OR 0.90 [0.72-1.14]), mRS 0-1 outcome (34.2\% vs. 33.7\%; adjusted OR 1.27 [0.94-1.72), mRS 0-2 outcome (52.4\% vs. 52.9\%; adjusted OR 1.21 [0.90-1.63]), and in-hospital mortality (6.1\% vs. 4.2\%; adjusted OR 1.19 [0.71-2.01]).
Correspondence: Keun-Sik Hong Department of Neurology, Ilsan Paik Hospital, Inje University, 170 Juhwa-ro, Ilsanseo-gu, Goyang 10380, Korea Tel: +82-31-910-7680 Fax: +82-31-910-7368 E-mail:nrhks@paik.ac.kr

Received: March 20. 2016

Revised: July 6, 2016

Accepted: July 7, 2016

*Jay Chol Choi and Ji Sung Lee contributed equally to the manuscript as the first author.

This study was supported from the Korea Healthcare Technology REtD Project, Ministry of Health, Republic of Korea (HI10C2020).

The authors have no financial conflicts of interest. 
Conclusions Despite an increased risk of SICH, prestroke antiplatelet users compared to non-users had comparable functional outcomes and in-hospital mortality with IV-TPA therapy. Our results support the use of IV-TPA in eligible patients taking antiplatelet therapy before their stroke onset.

Keywords Stroke; Thrombolytic therapy; Platelet aggregation inhibitors; Outcome assessment

\section{Introduction}

Intravenous tissue plasminogen activator (IV-TPA) improves outcomes of patients with acute ischemic stroke, but has the risk of symptomatic intracranial hemorrhage (SICH). IV-TPA therapy is not recommended in patients with current use of warfarin therapy with internationalized normal range $>1.7$ or use of nonvitamin $\mathrm{K}$ antagonist oral anticoagulants, but prestroke antiplatelet use is not a formal contraindication despite of potential increased risk of SICH.

In a recent meta-analysis and a large observational study, among patients treated with IV-TPA, prestroke antiplatelet therapy was associated with a higher risk of $\mathrm{SICH}$, but the effects on functional outcome had shown conflicting results. ${ }^{1-11}$ However, the results were largely driven by data from Caucasian populations. Asian populations are likely to have a higher risk of $\mathrm{SICH}$ with IV-TPA therapy, ${ }^{12}$ but there has been limited data on the effect of prestroke antiplatelet use on the SICH risk from Asian populations. $^{13,14}$

In Korea, among acute ischemic stroke patients, 29.2\% were taking antiplatelet therapy within 7 days at the time of stroke onset. ${ }^{15}$ Currently, the Korean guidelines do not preclude IV-TPA therapy for eligible patients who are taking prestroke antiplatelet agents, ${ }^{16}$ but no study has systematically explored the effect of prestroke antiplatelet therapy on the risk of $\mathrm{SICH}$ among patients treated with IV-TPA. Using data from a large prospective stroke registry, this study aimed to assess the effect of prestroke antiplatelet use on the risk of $\mathrm{SICH}$ and functional outcomes in Korean patients treated with IV-TPA.

\section{Methods}

\section{Database}

In this study, we used data of the Clinical Research Center for Stroke-5 (CRCS-5) registry, which is a prospective registry of patients with acute ischemic stroke admitted to 15 academic centers in Korea. The CRCS- 5 prospective registry was approved by the Institutional Review Boards of all participating centers (http://www.stroke-crc.or.kr). Detailed information on the CRCS5 registry have been published previously. ${ }^{15,17}$ Because the purposes of the registry were to monitor and improve the quality of stroke care in Korea and a computer-assisted de-identification system ensured the anonymity of individual patients during enrollment, the informed consent from individual patients or their legally authorized representatives was waived by the relevant institutional review boards. However, this study was additionally approved by the institutional review board of Inje University IIsan Paik Hospital where the principal investigator of this study is affiliated.

\section{Study population}

Of 27,511 patients with acute ischemic stroke enrolled in the CRCS-5 registry between October 2009 and November 2014, 2,611 patients were treated with IV-TPA. We excluded the following patients; (1) missing information on the prestroke antiplatelet use, (2) prestroke warfarin therapy with international normalized ratio (INR) $>1.7$ or non-vitamin $\mathrm{K}$ antagonist oral anticoagulants, (3) endovascular recanalization therapy added to IV-TPA therapy, (4) prestroke modified Rankin Scale (mRS) score $>1$, (5) IV-TPA treatment at outside hospital, (6) IV-TPA treatment beyond 4.5 hours, and (7) unavailability of discharge mRS score.

\section{Study variables and outcomes}

Prestroke antiplatelet use was defined as taking antiplatelet within 7 days before the onset of index ischemic stroke. At the inception of the CRCS-5 registry on April 2008, we prospectively compiled data of the use of prestroke antiplatelet including aspirin, clopidogrel, cliostazol, triflusal, dipyridamole, ticlopidine, other antiplatelet, and any combination of antiplatelets. Additionally, we prospectively collected data regarding demographics, vascular risk factors, prestroke medications, initial stroke severity and ischemic stroke subtypes, vital signs, laboratory findings, $\mathrm{SICH}$, and discharge mRS score. The primary safety outcome was $\mathrm{SICH}$, which was defined as any hemorrhagic transformation associated with National Institutes of Health Stroke Scale (NIHSS) score worsening $\geq 4$ points. Secondary outcomes were discharge $\mathrm{mRS}$ score and in-hospital mortality.

\section{Statistical analysis}

For missing variables, variables with missing data of more than $10 \%$ of total observation were excluded from the analysis. Sim- 
ple imputation with median values was used for continuous variables with missing data of less than $10 \%$ of the total observations. For descriptive statistics, continuous variables were summarized as means (standard deviations) or median (interquartile range), and categorical variables as numbers with percentages. Baseline characteristics between prestroke antiplatelet users and non-users were compared with Student $t$-test for continuous variables with a normal distribution, Wilcoxon rank-sum test for continuous variables without a normal distribution, or the chi-square test for categorical variables.

To compare the risks of SICH and mortality between the two groups, we used Chi-square test or Fisher's exact test for unadjusted analysis and binary logistic regression analysis for covariates adjustment. To estimate absolute difference of SICH between two groups after multivariable adjustment, we used the method described by Ciolino et al. ${ }^{18}$ For mRS outcomes, we compared the overall distribution of discharge mRS scores and dichotomized mRS outcomes (mRS 0-1 vs. 2-6 and mRS 0-2 vs. $3-6)$ between the two groups. The odds ratio (OR) with 95\% confidence interval $(\mathrm{Cl})$ was calculated to estimate the probability of favorable shift on the mRS score with prestroke antiplatelet use. The Cochran-Mantel-Haenszel test was used for unadjusted

Table 1. Characteristics of patients

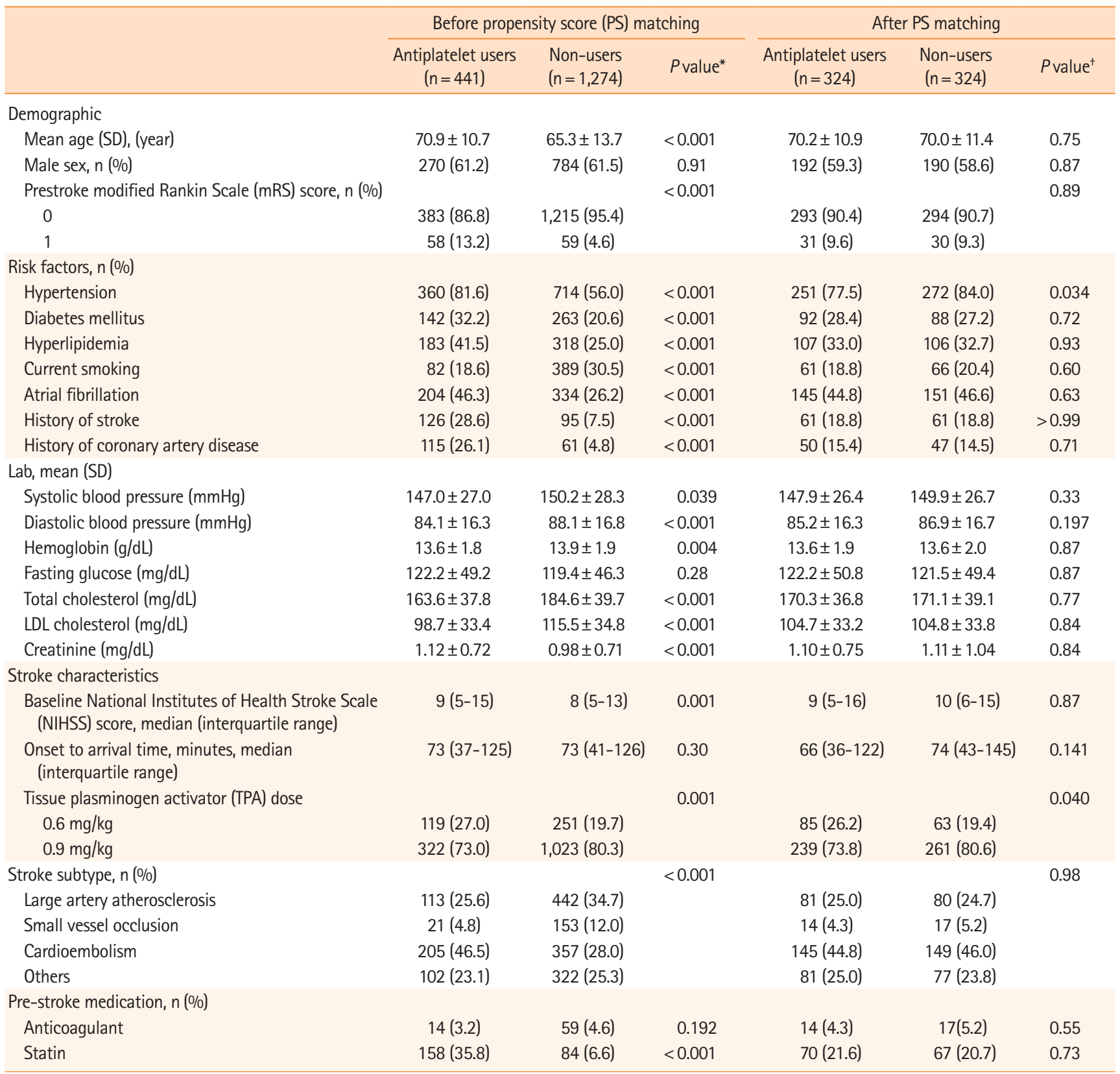

${ }^{*} P$ values are calculated by Pearson chi-square test, Fisher's exact test, Student's $t$-test or Wilcoxon rank sum test as appropriate; ${ }^{+} P$ values are calculated by conditional logistic regression. 
shift analysis, and the ordinal logistic regression analysis was used to adjust covariates.

In addition to multivariable analyses, we performed propensity score (PS)-matched analysis because the baseline characteristics of patients taking prestroke antiplatelet and those without prestroke antiplatelet were expected to differ significantly. To estimate PS, the binary logistic regression with all relevant characteristics related to the prestroke antiplatelet was used. The PS model included all the variables shown in Table 1 except for onset to arrival time, and TPA dose. Greedy-matching algorithm was employed with width of calipers between 0.2 and 0.6 standard deviations of the logit of the estimated PS. To check the baseline imbalances between the two groups after the PS matching, the absolute standardized difference (ASD) was computed for each covariate. We performed outcome analysis using ordinal or logistic regression analysis based on the generalized estimation equation method after the PS matching. Covariates with an ASD >0.1 were further adjusted in PS-matched analysis. However, we included age, sex, initial NIHSS score, onset to treatment time, and TPA dose in the final model as clinically important covariates regardless of their ASD values. As a sensitivity test, we repeated the same analyses with $\mathrm{mRS}$ outcome at 3 months after the stroke as a dependent variable. In all analyses, a $P$ value $<0.05$ was considered statistically significant. SAS computer software (Version 9.3, SAS Institute, Cary, NC, USA) was used for the statistical analyses.

\section{Results}

\section{Patients}

Of 2,611 patients treated with IV-TPA, we excluded 896 patients in the following order: (1) missing information on the prestroke antiplatelet use (16 patients), (2) prestroke warfarin therapy with INR $>1.7$ or non-vitamin $\mathrm{K}$ antagonist oral anticoagulants (4 patients), (3) prestroke mRS score $>1$ (420 patients), (4) IV-TPA treatment at outside hospital (325 patients), (5) treated with IV-TPA beyond 4.5 hours (128 patients), and (6) unavailability of discharge mRS score (3 patients) (Figure 1). As a result, 1,715 patients were included in this study, and 441 (25.7\%) were prestroke antiplatelet users. Of them, 353 patients were taking single antiplatelet therapy (aspirin, 279 patients; clopidogreal, 59 patients; 15 other monotherapy), 87 patients were taking dual antiplatelet therapy (aspirin plus clopidogrel, 69 patients; other dual therapy, 19 patients) and one patient was taking triple antiplatelet therapy. Table 1 presents demographic and clinical characteristics of prestroke antiplatelet users and non-users before and after PS matching. Before PS matching, prestroke antiplatelet users versus non-users were more likely to be older, to have

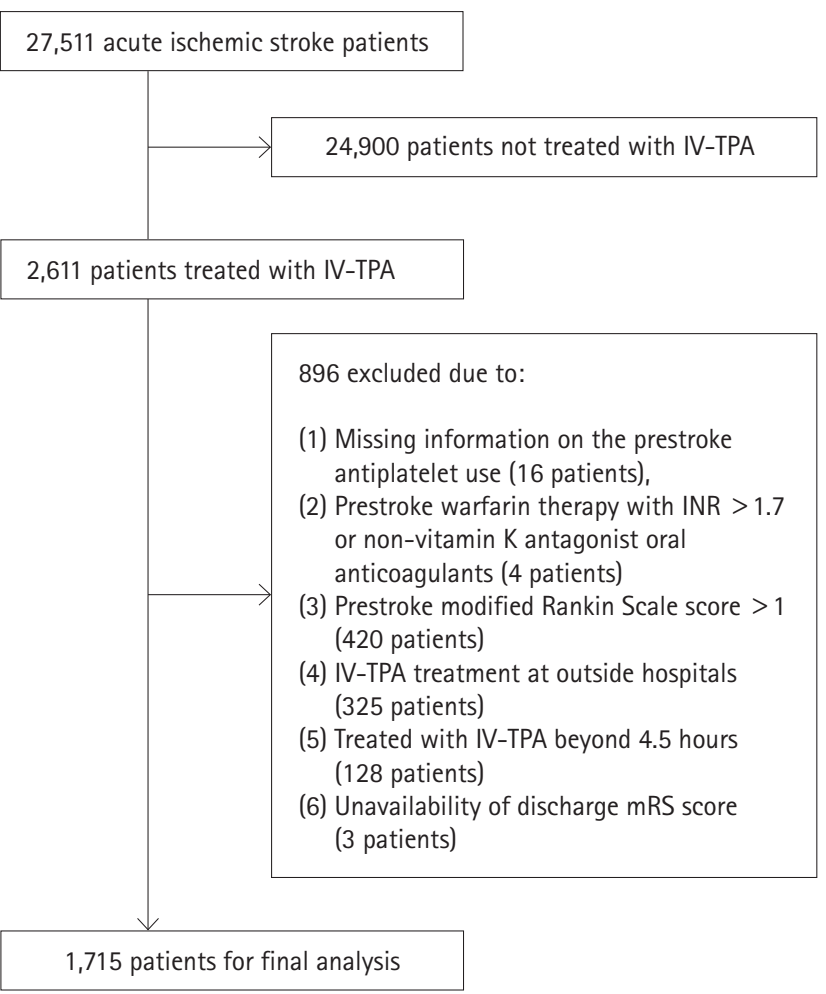

Figure 1. Flow diagram identifying eligible patients. IV-TPA, intravenous tissue plasminogen activator; INR, international normalized ratio; $\mathrm{mRS}$, modified Rankin Scale.

prestroke disability, hypertension, diabetes, dyslipidemia, atrial fibrillation, prior stroke, and coronary heart disease, to take statins, and to have lower levels of blood pressure, total and low-density lipoprotein (LDL) cholesterol, and hemoglobin. They were also more likely to have higher NIHSS score at baseline, and to have stroke subtype of cardioembolism. After PS matching, except for hypertension and TPA dose, the baseline characteristics were well balanced between the two groups (Supplemental Figure 1). Among 324 patients treated with prestroke antiplatelet therapy, $273(84.3 \%)$ were taking single antiplatelet therapy and 51 (15.7\%) were taking dual antiplatelet therapy.

\section{Symptomatic intracranial hemorrhage}

Overall, SICH occurred in 64 patients (3.7\%) after IV-TPA therapy. Before PS matching, prestroke antiplatelet users versus nonusers had a higher rate of SICH before adjustment (5.9\% vs. 3.0\%; unadjusted OR 2.04 [1.22-3.40]), and the increased risk remained significant after multivariable adjustment (OR 1.79 [1.05-3.04]). The estimated absolute difference after adjustment was 1.8\%. In particular, prestroke dual antiplatelet use was associated with a greater risk of SICH (unadjusted OR 3.29 [1.287.48]) compared to single antiplatelet use (unadjusted OR 1.75 [0.93-3.19]). 
In PS matching analysis, there was a trend of increased risk of $\mathrm{SICH}$ with prestroke antiplatelet use, but it did not reach statistical significance (OR 1.94 [0.96-3.93]) (Table 2).

In subgroup analyses, the increased risk of $\mathrm{SICH}$ with prestroke antiplatelet use did not differ among subgroups stratified by age, sex, diabetes mellitus, IV-TPA treatment window, TPA dose, baseline NIHSS score, and prestroke statin use (Table 3 and Figure 2).

\section{Disability outcomes}

At discharge the two groups did not differ in mRS distribution (adjusted OR 0.90 [0.72-1.14]; adjusted OR after PS matching 0.94 [0.71-1.23]), mRS 0-1 outcome (34.2\% vs. 33.7\%; adjusted OR 1.27 [0.94-1.14]; adjusted OR after PS matching 1.24 [0.871.79]), mRS 0-2 outcome (52.4\% vs. 52.9\%; adjusted OR 1.21
[0.90-1.63]; adjusted OR after PS matching 1.20 [0.84-1.70]), and in-hospital mortality (6.1\% vs. 4.2\%; adjusted OR 1.19 [0.712.01]; adjusted OR after PS matching 1.26 [0.60-2.65]) in both multivariable and PS matching analyses (Table 4 and Figure 3 ). The mRS score at 3 months after the stroke was available for 1,432 patients (83.5\%), and prestroke antiplatelet users versus non-users had comparable mRS distribution or dichotomized mRS outcomes in multivariable and PS matching analysis (Table 5).

\section{Discussion}

Using a nationwide prospective stroke registry database, we found that, among patients treated with IV-TPA, the use of prestroke antiplatelet was associated with almost two-fold in-

Table 2. Risks of symptomatic intracranial hemorrhage (SICH) and in-hospital mortality with prestroke antiplatelet use

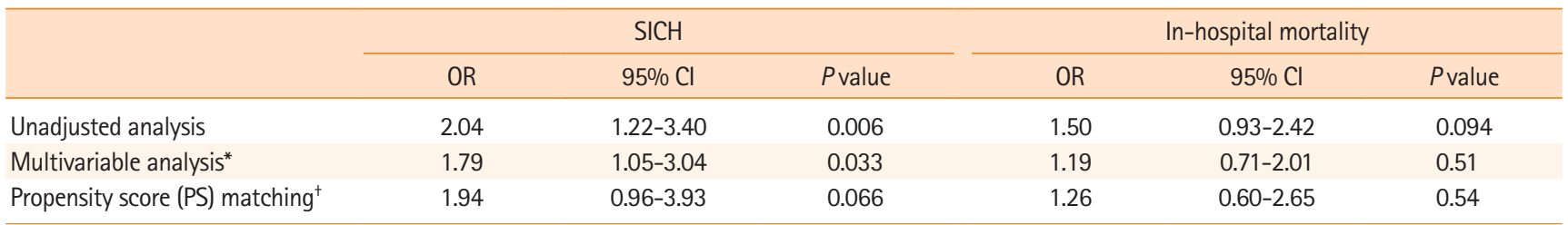

$\mathrm{OR}$, odds ratio; $\mathrm{Cl}$, confidence interval.

${ }^{*}$ Adjusted for age, sex, initial National Institutes of Health Stroke Scale (NIHSS) score, onset to treatment time, history of diabetes, and TPA dose; ${ }^{+}$PS matching samples included 324 pairs and were adjusted for age, sex, initial NIHSS score, onset to treatment time, history of diabetes, diastolic blood pressure and TPA dose.

Table 3. Risks of symptomatic intracranial hemorrhage (SICH) with prestroke antiplatelet therapy

\begin{tabular}{|c|c|c|c|c|c|c|c|}
\hline \multirow{2}{*}{ Characteristics } & \multirow{2}{*}{$\mathrm{N}$} & \multicolumn{5}{|c|}{ SICH rate, $n(\%)$} & \multirow{2}{*}{$\begin{array}{c}\text { Interaction } \\
P \text { value }^{*}\end{array}$} \\
\hline & & Antiplatelet users & Non-users & Crude OR & $95 \% \mathrm{Cl}$ & $P$ value & \\
\hline \multicolumn{8}{|l|}{ Age } \\
\hline$>80$ years & 276 & $5(5.2)$ & $10(5.6)$ & 0.93 & $0.24-3.11$ & 0.90 & 0.13 \\
\hline$\leq 80$ years & 1,439 & $21(6.1)$ & $28(2.6)$ & 2.47 & $1.31-4.57$ & 0.002 & \\
\hline \multicolumn{8}{|l|}{ Sex } \\
\hline Male & 1,054 & $16(5.9)$ & $18(2.3)$ & 2.68 & $1.26-5.66$ & 0.005 & 0.25 \\
\hline Female & 661 & $10(5.8)$ & $20(4.1)$ & 1.46 & $0.60-3.35$ & 0.34 & \\
\hline \multicolumn{8}{|c|}{ Diabetes mellitus } \\
\hline Yes & 1,310 & $17(5.7)$ & $32(3.2)$ & 1.84 & $1.01-3.37$ & 0.047 & 0.47 \\
\hline No & 405 & $9(6.3)$ & $6(2.3)$ & 2.90 & $1.01-8.32$ & 0.048 & \\
\hline \multicolumn{8}{|c|}{$\begin{array}{l}\text { Baseline National Institutes of Health } \\
\text { Stroke Scale (NIHSS) score }\end{array}$} \\
\hline$\leq 8$ & 870 & $6(3.1)$ & $9(1.3)$ & 2.35 & $0.68-7.49$ & 0.109 & 0.62 \\
\hline$>8$ & 845 & $20(8.1)$ & $29(4.8)$ & 1.74 & $0.91-3.26$ & 0.066 & \\
\hline \multicolumn{8}{|c|}{ Onset to treatment } \\
\hline$\leq 3$ hours & 1,414 & $22(5.9)$ & $29(2.8)$ & 2.21 & $1.19-4.04$ & 0.006 & 0.55 \\
\hline 3-4.5 hours & 301 & $4(5.6)$ & $9(3.9)$ & 1.46 & $0.32-5.45$ & 0.54 & \\
\hline \multicolumn{8}{|c|}{ Tissue plasminogen activator (TPA) dose } \\
\hline $0.6 \mathrm{mg} / \mathrm{kg}$ & 370 & $5(4.2)$ & $7(2.8)$ & 1.53 & $0.37-5.73$ & 0.48 & 0.57 \\
\hline $0.9 \mathrm{mg} / \mathrm{kg}$ & 1,345 & $21(6.5)$ & $31(3.0)$ & 2.23 & $1.26-3.94$ & 0.006 & \\
\hline \multicolumn{8}{|l|}{ Prestroke statin } \\
\hline Yes & 1,473 & $17(6.0)$ & $36(3.0)$ & 2.05 & $1.13-3.70$ & 0.012 & 0.82 \\
\hline No & 242 & $9(5.7)$ & $2(2.4)$ & 2.48 & $0.52-11.74$ & 0.25 & \\
\hline
\end{tabular}

$\mathrm{OR}$, odds ratio; $\mathrm{Cl}$, confidence interval.

*Interaction effect between prestroke antiplatelet use and subgroups. 
creased risk of SICH compared with no prior antiplatelet use. Despite the increased risk of SICH with prestroke antiplatelet use, an absolute increase of SICH risk was not substantial and therefore it did not translate into an increased risk of in-hospital mortality nor worsening of functional outcome. These findings strongly support the current stroke guidelines that do not preclude patients taking antiplatelet agents from IV-TPA treatment.

Several large observational studies with appropriate adjustment methods and recent meta-analyses have largely found an

Age
$>80$ years
$\leq 80$ years
Sex
Male
Female
DM
Yes
No
Baseline NIHSS score
$\leq 8$
$>8$
Onset to treatment time
$\leq 3$ hours
$3-4.5$ hours
TPA dose
0.6 mg/kg
0.9 mg/kg
Prestroke statin
Yes
No

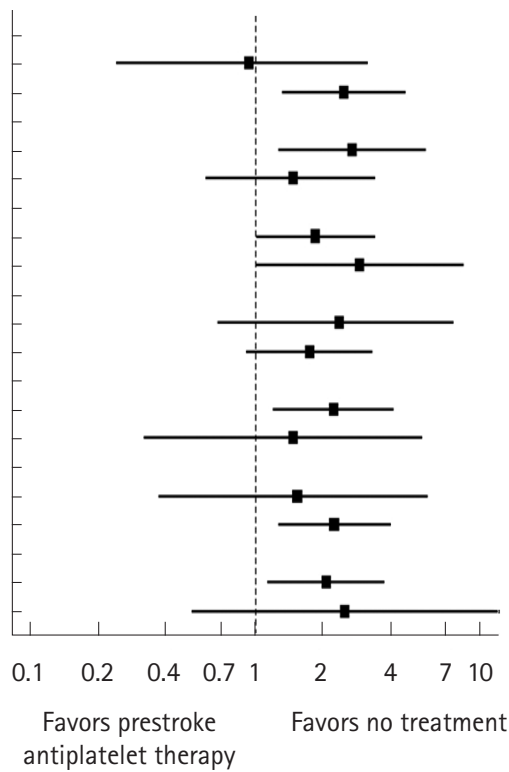

Figure 2. Prestroke antiplatelet therapy and risks of symptomatic intracranial hemorrhage. association between prestroke antiplatelet use and increased risk of SICH. ${ }^{1,2,419}$ Regarding disability and mortality outcomes, exploratory analysis of Safe Implementation of Thrombolysis in Stroke-Monitoring (SITS-MOST) data showed that antiplatelet other than aspirin was associated with increased risk of mortality, but disability outcome was not affected by prestroke antiplatelet use. ${ }^{19}$ In contrast, a subsequent study analyzing a larger

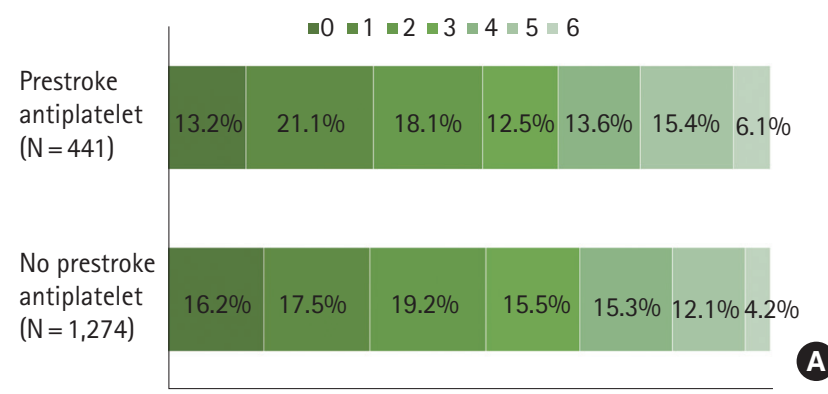

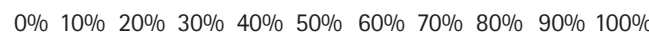

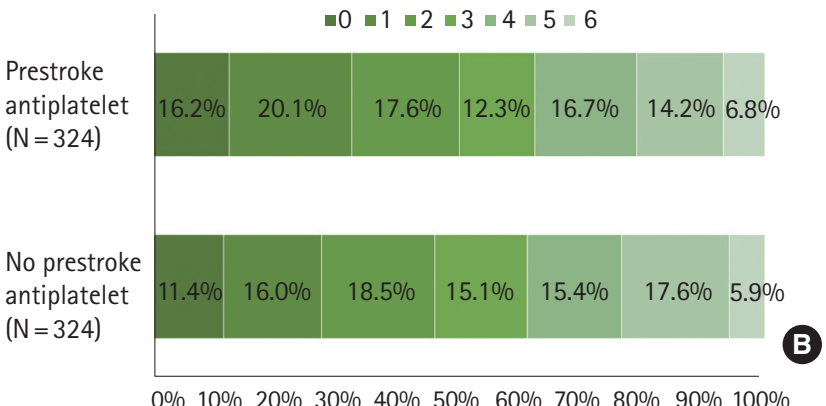

Figure 3. Functional outcomes at discharge by prestroke antiplatelet use before (A) and after propensity score matching (B)

Table 4. Effectiveness of prestroke antiplatelet use on discharge modified Rankin Scale (mRS) outcome

\begin{tabular}{|c|c|c|c|c|c|c|c|c|c|}
\hline & \multicolumn{3}{|c|}{ Shift analysis } & \multicolumn{3}{|c|}{ mRS 0-1 proportion } & \multicolumn{3}{|c|}{ mRS 0-2 proportion } \\
\hline & $\mathrm{OR}^{*}$ & $95 \% \mathrm{Cl}$ & $P$ value & $\mathrm{OR}$ & $95 \% \mathrm{Cl}$ & $P$ value & $\mathrm{OR}$ & $95 \% \mathrm{Cl}$ & $P$ value \\
\hline Unadjusted analysis & 1.13 & $0.93-1.37$ & 0.21 & 1.03 & $0.82-1.29$ & 0.83 & 0.98 & $0.79-1.22$ & 0.85 \\
\hline Multivariable analysis $^{+}$ & 0.90 & $0.72-1.14$ & 0.38 & 1.27 & 0.94-1.72 & 0.118 & 1.21 & $0.90-1.63$ & 0.20 \\
\hline Propensity score (PS)-matched analysis ${ }^{\ddagger}$ & 0.94 & $0.71-1.23$ & 0.64 & 1.24 & $0.87-1.79$ & 0.24 & 1.20 & $0.84-1.70$ & 0.32 \\
\hline
\end{tabular}

$\mathrm{OR}$, odds ratio; $\mathrm{Cl}$, confidence interval.

${ }^{*}$ Odd ratios for achieving a favorable shifting of one level on the mRS score; ${ }^{+}$Adjusted for age, sex, initial National Institutes of Health Stroke Scale (NIHSS), onset to treatment time, tissue plasminogen activator (TPA) dose and variables with $P<0.1$ in the Table $1 ;{ }^{*}$ PS matching samples included 324 pairs and were adjusted for age, sex, initial NIHSS score, onset to treatment time, history of diabetes, diastolic blood pressure and TPA dose.

Table 5. Effectiveness of tissue plasminogen activator (TPA) on modified Rankin Scale (mRS) outcomes at 3 months after the stroke

\begin{tabular}{|c|c|c|c|c|c|c|c|c|c|}
\hline & \multicolumn{3}{|c|}{ Shift analysis } & \multicolumn{3}{|c|}{ mRS 0-1 proportion } & \multicolumn{3}{|c|}{ mRS 0-2 proportion } \\
\hline & $O R^{*}$ & $95 \% \mathrm{Cl}$ & $P$ value & $\mathrm{OR}$ & $95 \% \mathrm{Cl}$ & $P$ value & $\mathrm{OR}$ & $95 \% \mathrm{Cl}$ & $P$ value \\
\hline Unadjusted analysis & 1.30 & $1.06-1.60$ & 0.013 & 0.82 & $0.65-1.05$ & 0.110 & 0.75 & $0.59-0.95$ & 0.0163 \\
\hline Multivariable analysis ${ }^{\dagger}$ & 1.13 & $0.87-1.46$ & 0.36 & 0.92 & $0.66-1.28$ & 0.61 & 0.83 & $0.59-1.17$ & 0.28 \\
\hline Propensity score (PS)-matched analysis $^{*}$ & 1.04 & $0.76-1.41$ & 0.82 & 1.07 & $0.72-1.60$ & 0.74 & 1.05 & $0.70-1.56$ & 0.83 \\
\hline
\end{tabular}

$\mathrm{OR}$, odds ratio; $\mathrm{Cl}$, confidence interval.

${ }^{*}$ Odd ratios for achieving a favorable shifting of one level on the mRS score; ${ }^{+}$Adjusted for age, sex, initial National Institutes of Health Stroke Scale (NIHSS), onset to treatment time, TPA dose and variables with $P<0.1$ in the Table $1 ;{ }^{*} P S$ matching samples included 324 pairs and were adjusted for age, sex, initial NIHSS score, onset to treatment time, history of diabetes, diastolic blood pressure and TPA dose. 
dataset from Safe Implementation of Treatments in Stroke International Stroke Thrombolysis Register (SITS-ISTR) showed no increased risk in mortality and poor outcome of mRS 3-6 with prestroke antiplatelet use, ${ }^{2}$ and a meta-analysis involving 19,453 patients also showed no clear association between prestroke antiplatelet use and functional outcome. ${ }^{1}$ However, in a recent observational study of the Get With the Guidelines (GWTG)-Stroke registry enrolling approximately 85,000 patients, despite an increased risk of $\mathrm{SICH}$, prestroke antiplatelet use was associated with a better functional outcome and did not increase the mortality. ${ }^{6}$ The absolute increase in the $\mathrm{SICH}$ risk with prestroke antiplatelet use was relatively small in prior studies (unadjusted absolute increase of $1.4 \% \%^{2}$ and adjusted absolute increase of $0.68 \%{ }^{6}$ ) and our study (adjusted absolute increase of 1.8\%), which might account for no substantial effect of SICH risk with prestroke antiplatelet use on clinical outcomes across studies.

Earlier studies suggested a greater risk of $\mathrm{SICH}$ with dual antiplatelet therapy compared with monotherapy. ${ }^{1,2}$ The higher risk of SICH with dual antiplatelet use compared to single antiplatelet use was reproduced in a large cohort study of the GWTGStroke registry: adjusted OR with any antiplatelet therapy, 1.18 (1.10-1.28), number needed to harm (NNH)147; adjusted OR with dual therapy, 1.47 (1.16-1.86), NNH 60. ${ }^{6}$ Another Chinese nationwide prospective stroke registry of IV-TPA therapy also reported the risk of SICH by prestroke antiplatelet status; among 1,108 patients 157 patients (14.2\%) were on antiplatelet therapy, and only 14 patients had aspirin and clopidogrel dual therapy. They found a substantial increase in the SICH risk with dual antiplatelet therapy (aspirin and clopidogrel) (adjusted OR 5.92; 95\% Cl 1.29-27.06) but not with any antiplatelet or aspirin monotherapy. ${ }^{13}$ Our study also showed that patients taking dual antiplatelet therapy seemed to exhibit a higher risk of $\mathrm{SICH}$ compared to those with single antiplatelet therapy. Thereby, physicians, patients, and surrogates need to be informed about the possible higher SICH risk of IV-TPA therapy in patients taking dual antiplatelet therapy.

Because of limited data, we were not able to explore the differential effect of individual antiplatelet regimens on the $\mathrm{SICH}$ risk. In a Taiwanese study, the use of clopidogrel or ticlopidine was associated with a greater risk of $\mathrm{SICH}$ in univariate analysis (OR 12.56, 95\% $\mathrm{Cl} 3.13-50.38$ ), and the increased risk remained significant even after adjustment. ${ }^{14}$ However, in the GWTGStroke registry study, the risk of $\mathrm{SICH}$ was not increased with prestroke clopidogrel monotherapy (adjusted OR 0.85 [0.651.12]), but it was increased with prestroke aspirin monotherapy (adjusted OR 1.19 [1.06-1.34]). ${ }^{6}$ Whether there is a racial disparity in the different antiplatelet effect on the SICH risk would be of interest as future investigation.
Our study has several limitations. First, this is an observational study that is at high risk of bias. To overcome the limitation, we conducted PS matching analysis in addition to multivariable analysis. Although the increased risk of $\mathrm{SICH}$ with prestroke antiplatelet use was not statistically significant with the PS matching analysis, there was a strong trend and the magnitude of $\mathrm{OR}$ was similar to that with the multivariable analysis. However, even with the PS matching analysis, unmeasured confounders could potentially affect outcome comparison. Second, limited patient numbers and events did not allow adjusted analysis for effects of different antiplatelet regimens on outcomes. Future studies using collaborative Asian multinational registry would be needed to investigate whether Asians are at greater risk of developing SICH following IV-TPA especially with thienopyridine antiplatelet agents or dual antiplatelet therapy than Caucasians. Third, prestroke antiplatlet use might affect functional outcome after stroke by enhancing recanalization rate. ${ }^{20}$ Unfortunately, we do not have information on the recanalization state for approximately 50\% of the patients and therefore the effect of prestroke antiplatelet on functional outcomes by the recanalization status could not be investigated. Finally, we do not have information on the last timing and dose of each antiplatelet agent, which might affect the outcome. In addition, we did not centrally adjudicate $\mathrm{SICH}$ for this study and we did not assess systemic bleeding complications other than SICH.

\section{Conclusions}

Korean patients with prestroke antiplatelet had an increased risk of $\mathrm{SICH}$, but had comparable functional outcomes and inhospital mortality following IV-TPA. Our findings along with earlier studies indicate that patients taking antiplatelet therapy should not be excluded from IV-TPA therapy given that similar net benefits could be achieved as in patients who are not taking any antiplatelet agent.

\section{References}

1. Pan $X$, Zhu Y, Zheng D, Liu Y, Yu F, Yang J. Prior antiplatelet agent use and outcomes after intravenous thrombolysis with recombinant tissue plasminogen activator in acute ischemic stroke: a meta-analysis of cohort studies and randomized controlled trials. Int J Stroke 2015;10:317-323.

2. Diedler J, Ahmed N, Sykora M, Uyttenboogaart M, Overgaard K, Luijckx GJ, et al. Safety of intravenous thrombolysis for acute ischemic stroke in patients receiving antiplatelet therapy at stroke onset. Stroke 2010;41:288-294.

3. Mazya M, Egido JA, Ford GA, Lees KR, Mikulik R, Toni $D$, et al. 
Predicting the risk of symptomatic intracerebral hemorrhage in ischemic stroke treated with intravenous alteplase: safe Implementation of Treatments in Stroke (SITS) symptomatic intracerebral hemorrhage risk score. Stroke 2012;43:1524-1531.

4. Whiteley WN, Slot KB, Fernandes P, Sandercock P, Wardlaw J. Risk factors for intracranial hemorrhage in acute ischemic stroke patients treated with recombinant tissue plasminogen activator: a systematic review and meta-analysis of 55 studies. Stroke 2012;43:2904-2909.

5. Whiteley WN, Thompson D, Murray G, Cohen G, Lindley RI, Wardlaw J, et al. Targeting recombinant tissue-type plasminogen activator in acute ischemic stroke based on risk of intracranial hemorrhage or poor functional outcome: an analysis of the third international stroke trial. Stroke 2014;45:1000-1006.

6. Xian Y, Federspiel JJ, Grau-Sepulveda M, Hernandez AF, Schwamm LH, Bhatt DL, et al. Risks and benefits associated with prestroke antiplatelet therapy among patients with acute ischemic stroke treated with intravenous tissue plasminogen activator. JAMA Neuro/ 2016;73:50-59.

7. Bluhmki E, Chamorro A, Dávalos A, Machnig T, Sauce C, Wahlgren $\mathrm{N}$, et al. Stroke treatment with alteplase given 3.0-4.5 h after onset of acute ischaemic stroke (ECASS III): additional outcomes and subgroup analysis of a randomised controlled trial. Lancet Neurol 2009;8:1095-1102.

8. Cucchiara B, Kasner SE, Tanne D, Levine SR, Demchuk A, Messe

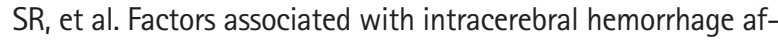
ter thrombolytic therapy for ischemic stroke: pooled analysis of placebo data from the Stroke-Acute Ischemic NXY Treatment (SAINT) I and SAINT II Trials. Stroke 2009;40:3067-3072.

9. Ibrahim MM, Sebastian J, Hussain M, Al-Hussain F, Uchino K, Molina $C_{1}$ et al. Does current oral antiplatelet agent or subtherapeutic anticoagulation use have an effect on tissue-plasminogen-activator-mediated recanalization rate in patients with acute ischemic stroke? Cerebrovasc Dis 2010;30:508-513.

10. Larrue V, von Kummer RR, Müller A, Bluhmki E. Risk factors for severe hemorrhagic transformation in ischemic stroke patients treated with recombinant tissue plasminogen activator: a secondary analysis of the European-Australasian Acute Stroke Study (ECASS II). Stroke 2001;32:438-441.

11. Tanne D, Kasner SE, Demchuk AM, Koren-Morag N, Hanson S, Grond $M_{1}$ et al. Markers of increased risk of intracerebral hemorrhage after intravenous recombinant tissue plasminogen ac- tivator therapy for acute ischemic stroke in clinical practice: the Multicenter rt-PA Stroke Survey. Circulation 2002;105: 1679-1685.

12. Mehta RH, Cox M, Smith EE, Xian Y, Bhatt DL, Fonarow GC, et al. Race/Ethnic differences in the risk of hemorrhagic complications among patients with ischemic stroke receiving thrombolytic therapy. Stroke 2014;45:2263-2269.

13. Pan $Y$, Chen $Q$, Liao $X$, Zhao $X$, Wang $C$, Liu G, et al. Preexisting dual antiplatelet treatment increases the risk of post-thrombolysis intracranial hemorrhage in Chinese stroke patients. Neurol Res 2015;37:64-68.

14. Chao AC, Hsu HY, Chung CP, Liu CH, Chen $\mathrm{CH}$, Teng MM, et al. Outcomes of thrombolytic therapy for acute ischemic stroke in Chinese patients: the Taiwan Thrombolytic Therapy for Acute Ischemic Stroke (TT-AIS) study. Stroke 2010;41:885-890.

15. Kim BJ, Park JM, Kang K, Lee SJ, Ko Y, Kim JG, et al. Case characteristics, hyperacute treatment, and outcome information from the clinical research center for stroke-fifth division registry in South Korea. J Stroke 2015;17:38-53.

16. Cho KH, Ko SB, Kim DH, Park HK, Cho AH, Hong KS, et al. Focused update of Korean clinical practice guidelines for the thrombolysis in acute stroke management. Korean J Stroke 2012;14:95-105.

17. Kim BJ, Han MK, Park TH, Park SS, Lee KB, Lee BC, et al. Current status of acute stroke management in Korea: a report on a multicenter, comprehensive acute stroke registry. Int J Stroke 2014;9:514-518.

18. Ciolino JD, Martin RH, Zhao W, Jauch EC, Hill MD, Palesch YY. Covariate imbalance and adjustment for logistic regression analysis of clinical trial data. J Biopharm Stat 2013;23:13831402.

19. Wahlgren N, Ahmed N, Eriksson N, Aichner F, Bluhmki $E_{\text {, }}$ Dávalos $A$, et al. Multivariable analysis of outcome predictors and adjustment of main outcome results to baseline data profile in randomized controlled trials: Safe Implementation of Thrombolysis in Stroke-MOnitoring STudy (SITS-MOST). Stroke 2008;39:3316-3322.

20. Hallevi H, Grotta JC. Antiplatelet therapy and the risk of intracranial hemorrhage after intravenous tissue plasminogen activator therapy for acute ischemic stroke. Arch Neurol 2008;65: 575-576. 


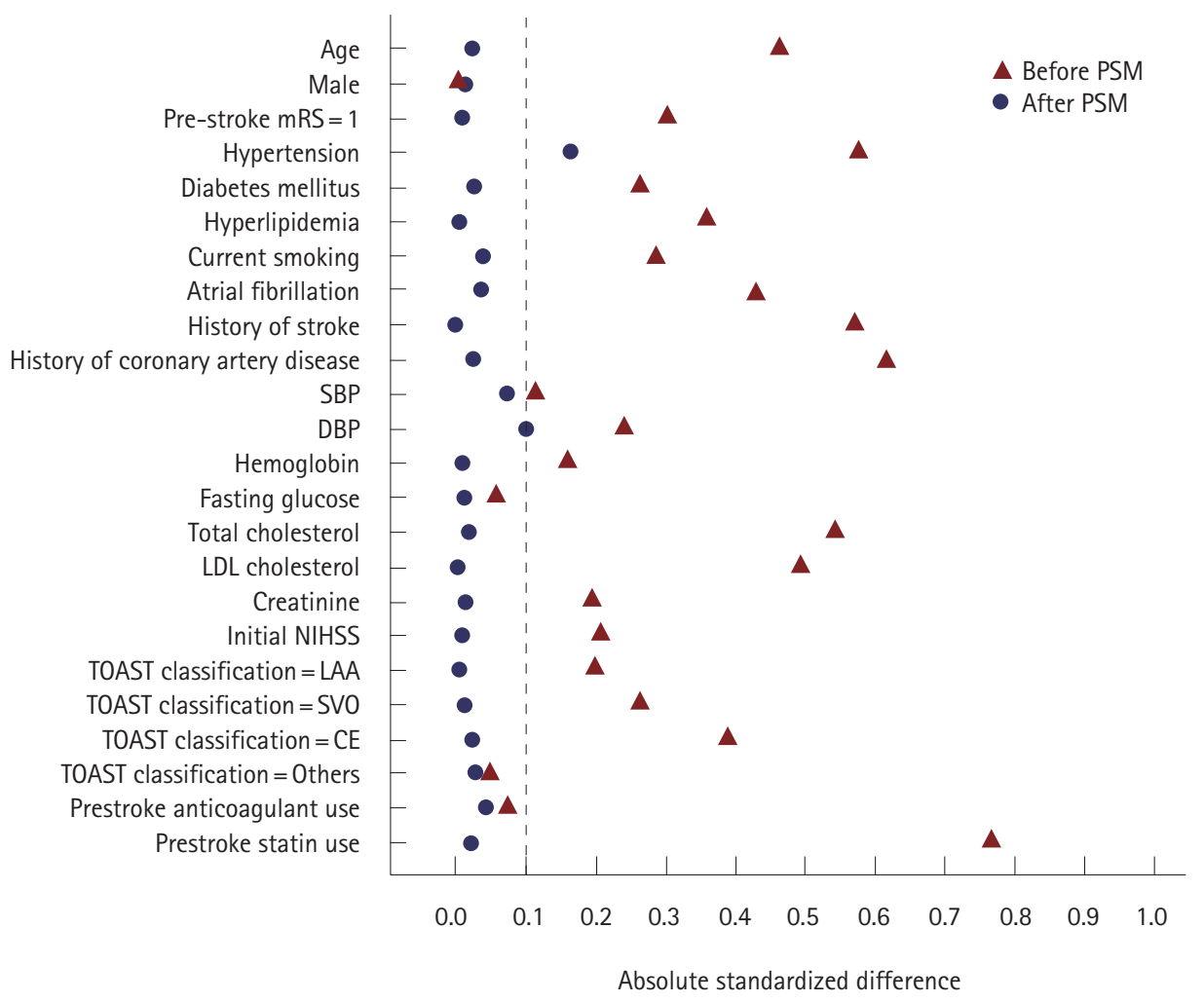

Supplementary Figure 1. Absolute standardized difference of covariates before and after propensity score matching. mRS, modified Rankin Scale; SBP, systolic blood pressure; DBP, diastolic blood pressure; LDL, low-density lipoprotein; NIHSS, National Institutes of Health Stroke Scale; TOAST, Trial of Org 10172 in Acute Stroke Treatment; LAA, large-artery atherosclerosis; SVO, small-vessel occlusion; CE, cardioembolism. 